\title{
¿QUÉ ES LA ECONOMÍA? UNA DISCIPLINA POLÍTICA PARA EL MUNDO REAL*
}

James K. Galbraitha

" DOI: https://doi.org/10.18601/01245996.v24n46.02. Recepción: 15-082021, aceptación: 29-11-2021. Sugerencia de citación: Galbraith, James K. (2022). ¿Qué es la economía? Una disciplina política para el mundo real. Revista de Economía Institucional, 24(46), 3-25. Publicado originalmente en real-world esconomic review, 96, 67-81 E1 ensayo está dedicado a la memoria de Eugenia Correa Vásquez.

a Profesor en la Escuela Lyndon B. Johnson de Asuntos Públicos de la Universidad de Texas, [galbraith@mail.utexas.edu]. 


\section{¿Qué es la economía? Una disciplina política para el mundo real}

Resumen La economía es una disciplina política que trata los problemas de la organización social y del bien general, coevoluciona con las circunstancias y es históricamente contingente. $\mathrm{El}$ mundo al que se dirigen las políticas económicas es un sistema complejo, pero los economistas que buscan elaborar políticas apropiadas se guían necesariamente por simplificaciones y heurísticas. La pregunta que enfrenta la disciplina es qué tipo de simplificación se adapta mejor a la tarea. Este artículo argumenta que las generalizaciones, simplificaciones, heurísticas y principios apropiados se deben derivar del estudio del mundo real. Aunque pueden emplear herramientas matemáticas y aprovechar ideas del comportamiento de los sistemas matemáticos, estos son inadecuados, en especial cuando parten de los dogmas muertos de la corriente neoclásica: ex nibilo nibil fit.

Palabras clave: escuelas económicas, institucionalismo, teorías evolutivas, mundo real, complejidad, política económica, bienestar social; JEL: A11, B13, B15, C6, D6, H00

\section{What is economics? A policy discipline for the real world}

Abstract Economics is a policy discipline that deals with the problems of social organization and the general good. It co-evolves with circumstances, and it is historically contingent. The world to which economic policies are ultimately addressed is a complex system. Yet economists seeking to develop appropriate policies are necessarily guided by simplifications and heuristics. The question before the discipline is to decide what sort of simplification is best suited to the task. In the spirit of modern science, this paper argues that appropriate generalizations, simplifications, heuristics, and principles are to be derived from a study of the actual world. While these may deploy mathematical tools and draw on insights from the behavior of mathematical systems, the latter by themselves are inadequate, especially where they start from the dead dogmas of the neoclassical mainstream: ex nibilo nibil fit.

Keywords: economic schools, institutionalism, evolutionary theories, real-world, complexity, economic policy, social welfare; JEL: A11, B13, B15, C6, D6, H00

\section{Qual é a economia? Uma disciplina política para o mundo real}

Resumo Economia é uma disciplina política que lida com os problemas da organização.o bem geral e social, coevolui com as circunstâncias e é historicamente contingente. $\mathrm{O}$ mundo para o qual as políticas econômicas são direcionadas é um sistema complexo, mas os economistas que buscam fazer políticas adequadas são necessariamente guiados por simplificações e heurísticas. A questão que a disciplina enfrenta é que tipo de simplificação é mais adequada para a tarefa. Este artigo argumenta que generalizações, simplificações, heurísticas e princípios apropriados devem ser derivados do estudo do mundo real. Embora possam usar ferramentas matemáticas e tirar proveito de idéias do comportamento de sistemas matemáticos, estas são inadequadas, especialmente quando partem dos dogmas mortos da corrente neoclássica: ex nibilo nibil fit.

Palavras-chave: escolas econômicas, institucionalismo, teorias evolucionistas, mundo real, complexidade, política econômica, bem-estar social; JEL: A11, B13, B15, C6, D6, H00 


\begin{abstract}
Kepler se comprometió a trazar una curva a través de los lugares de Marte, y su mayor servicio a la ciencia fue dejar en la mente de los hombres que esto era lo que debian hacer para mejorar la astronomia; que no debian contentarse con preguntar si un sistema de epiciclos era mejor que otro, sino que debian apoyarse en las cifras y averiguar cuál en verdad era la curva.
\end{abstract}

Charles Sanders Peirce, 1877

Thara a economía es una disciplina política. Se ocupa de los problemas, grandes y pequeños, de la organización social y del bien general. Como tal, co-evoluciona con las circunstancias y es históricamente contingente. La aplicación de ideas económicas a problemas específicos en circunstancias específicas puede tener éxito o fracasar, y en este último caso, las personas que tienen ideas diferentes se suelen destacar.

E1 capitalismo es un sistema económico cuyas características y problemas han preocupado a los economistas desde el siglo XVIII. No es el único sistema de este tipo; antes del capitalismo hubo economistas que se remontan a Aristóteles. Y ha habido economistas en sistemas que compiten entre sí: el socialismo y el comunismo tenían sus propios economistas. Hoy es común hablar de "variedades del capitalismo" (Hall y Soskice, 2001), que promueven economistas con perspectivas y puntos de vista distintos. Los economistas y las teorías económicas son un subproducto del orden social que los engendra.

El mundo al que en últimas se dirigen las políticas económicas es un sistema complejo. Pero los economistas que buscan elaborar políticas apropiadas se guían necesariamente por simplificaciones y heurísticas. La pregunta que enfrenta la disciplina es qué tipo de simplificación se adapta mejor a la tarea. En el espíritu de C. S. Peirce y de la ciencia moderna, este artículo argumenta que las generalizaciones, simplificaciones, heurísticas y principios apropiados se deben derivar del estudio del mundo real. Aunque pueden emplear herramientas matemáticas y aprovechar ideas del comportamiento de los sistemas matemáticos, estos son en sí mismos inadecuados, en especial cuando parten de los dogmas muertos de la corriente neoclásica: ex nibilo nibil fit. Más adelante esbozamos los elementos de las estrategias de investigación que parecen adecuadas para un mundo económico complejo. Pero antes debemos hacer la distinción crítica entre la práctica de la economía como aquí se entiende y la disciplina académica que hoy se autodenomina economía. 


\section{EL DOGMA NEOCLÁSICO}

La economía académica contemporánea-ortodoxa, corriente principal, neoclásica- nació como reacción a una serie de giros radicales en la segunda mitad del siglo XIX. Entre ellos: a) el giro a la izquierda de la economía política clásica de David Ricardo a Karl Marx en la extensión lógica de la teoría del valor trabajo; b) la aplicación de Henry George de la doctrina del impuesto único de Ricardo a la tierra en Estados Unidos, a la que se opusieron los terratenientes; y c) las campañas de crédito fácil, bimetalistas y de plata gratuita del movimiento populista en las décadas de 1880 y 1890 , a las que se opusieron los banqueros (Frank, 2020). Detrás de esos movimientos económicos y políticos había un cambio aún más profundo en el carácter del pensamiento: el surgimiento del materialismo evolutivo y la aterradora comprensión de que el majestuoso y terrible aparato de la Naturaleza es producto de sistemas complejos autoorganizados regidos por un pequeño número de leyes físicas y biológicas irrevocables, incluidas la selección natural y la segunda ley de la termodinámica (Georgescu-Roegen, 1971).

Contra ese horror de cambios incesantes, tiempo irreversible y agitación potencial, contra la terrible idea de que las instituciones humanas son creadas por el hombre, mutables y en principio sujetas al control democrático, la economía neoclásica creó un templo al Dios de la Naturaleza, convenientemente domesticado bajo la apariencia de un mercado omnisciente, autorregulado y benigno. En este espejismo feliz, las antiguas nociones chinas de armonía celestial, adaptadas a la economía por François Quesnay (Davis, 1983), se transformaron en las tijeras de oferta y demanda de Alfred Marshall, y Leon Walras las generalizó al caso de $n$ mercancías en mercados perfectamente competitivos, cada uno equilibrado por precios flexibles a través de un subastador invisible. Después, Paul Samuelson (1947) echó el manto de J. Willard Gibbs sobre la formalización económica y se apropió indebidamente de la metáfora de la Mano Invisible de Adam Smith, que era demasiado apta para dejarla al uso prosaico que Smith le dio realmente ${ }^{1}$. Con el modelo de equilibrio general de Arrow-Debreu (1954) el sistema estaba casi completo, aceptando o impidiendo la

1 "Al preferir la industria nacional a la industria extranjera, solo persigue su propia seguridad; y al dirigir esa industria de modo que produzca el mayor valor, solo busca su propio beneficio, pero en este caso, como en muchos otros, es guiado por una mano invisible para promover un objetivo que no entraba en sus propósitos" (Smith, 1776). Roncaglia (2019, p. 177) señala que hay otras dos referencias a la frase en la obra de Smith, ninguna de las cuales apoya el significado que se suele atribuir a esa expresión. 
introducción de expectativas racionales y del agente representativo, lo que en última instancia llevó al equilibrio general computable (Scarf, 1973) y al modelo de equilibrio general estocástico dinámico.

E1 sistema neoclásico tenía dos atractivos. Primero, resonó con el apremio de todas las sociedades para justificarse invocando un propósito superior: la Voluntad de Dios, la misión civilizadora, el Destino Manifiesto, etc. Esa necesidad se agudiza cuando el principio organizador real de una cultura comercial es tan burdo como hacer dinero por sí mismo o el placer del consumo material. Segundo, el dogma dio una robusta respuesta ideológica primero al georgismo (Gaffney, 2007) y después al marxismo en el fétido clima intelectual de la Guerra Fría. Así se convirtió en puerta de entrada a una serie de sinecuras académicas de las que se excluía rigurosamente a los desviados; a pesar de que el trabajo práctico de hacer política económica siguió siendo realizado, en la mayoría de los países occidentales, por un puñado relativo de no neoclásicos no marxistas, en su mayoría seguidores de John Maynard Keynes, que de no ser así estaban condenados al ostracismo.

Desde el punto de vista de la hegemonía intelectual, lo más importante era el marco. Desafiando la máxima de Joseph Schumpeter (1942) de que el capitalismo es un sistema evolutivo, la economía neoclásica fijó las estructuras y los conceptos taxonómicos del campo de una vez por todas: interés propio racional, agentes representativos, empresas y hogares, capital y trabajo, precios y cantidades, ganancias y salarios, dinero neutral, tasas naturales de interés y desempleo, equilibrio general. Cualquier desviación de este marco se salía de los límites; por definición no era economía. La teoría era pura, y como la teoría pura no se aplicaba a nada, no podía evolucionar.

La economía ortodoxa dominante se enganchó así al profesor Pangloss y a su dogma atemporal de que todo es para mejor en el mejor de todos los mundos posibles, salvo cuando hay distorsiones, como preferencias interdependientes, bienes Giffen, bienes Veblen, monopolio, externalidades, bienes públicos, gasto público o impuestos, para no mencionar cualquier forma de incertidumbre no reducible a una distribución de probabilidad con varianza finita. En suma, la economía académica moderna adoptó el "modelo de un General de División moderno" en Piratas de Penzance de Gilbert y Sullivan². Su alcance se extiende a todas las situaciones imaginables, excepto a las que importan en el mundo real.

2 "Por mis conocimientos militares, aunque soy valiente y aventurero/ Solo he llegado hasta principios de siglo/ Pero aun así, en materia vegetal, animal y mineral/ soy el modelo de un General de División moderno”.

Revista de Economía Institucional, vol. 24 , n. ${ }^{\circ} 46$, Primer semestre/2022, Pp. 3-25 ISSN OI $24-5996 / \mathrm{E}-\mathrm{ISSN} 2346-2450$ 
En el mundo real, con la desaparición de los sistemas socialistas de Estado en la URSS y Europa del Este -aunque no en China- las doctrinas neoclásicas disfrutaron de un breve periodo de hegemonía real, captado en la célebre frase "el fin de la historia" (Fukuyama, 1992). En política, los esfuerzos por hacer que la realidad social parezca corresponder a los supuestos subyacentes de tipo ideal ya llevaban una década en marcha, y se aceleraron en un ambiente de triunfalismo. Desregulación, privatización, impuestos bajos, gobierno pequeño, libre comercio y dinero sano fueron las consignas de esta época, denotadas como neoliberalismo. En muy corto tiempo llevaron a la desindustrialización, al estancamiento, la desigualdad y la precariedad (Azmanova, 2020). Con la gran crisis financiera de 2007-2009 los dogmas quedaron expuestos y avergonzados: ¿una teoría que no tenía en cuenta el dinero ni el crédito, que de hecho no tenía sector bancario y carecía de cualquier concepto de fraude (Black, 2005), ¿`cómo podía explicar la mayor catástrofe financiera de todos los tiempos? Pero la inercia y la titularidad académica llevaron la economía neoclásica hacia la pandemia de 2020, momento en que se produjo un colapso adicional, posiblemente definitivo (Galbraith, 2020).

\section{ECONOMÍAS DEL COMPORTAMIENTO Y DE LA COMPLEJIDAD}

¿Qué ocupará el lugar de la economía neoclásica y su rama política neoliberal? No faltan candidatos, agrupados bajo la amplia bandera de heterodoxia económica. Algunas de estas doctrinas sucesoras -la economía conductual y la economía de la complejidad son ejemplos notables - toman la ortodoxia neoclásica como punto de partida. Por ello, se siguen definiendo a sí mismas en relación con esa ortodoxia. Otras evitaron por completo la atracción gravitacional o, como en el caso excepcional de Keynes, libraron una "larga lucha para escapar".

Los teóricos del comportamiento se apartan del neoclasicismo renunciando a los supuestos estrictos de racionalidad y maximización del comportamiento. Los teóricos de la complejidad exploran la dinámica de los agentes que interactúan y las funciones recursivas. Ambos logran cierta reputación académica manteniendo un estrecho diálogo con la corriente ortodoxa. Y solo rinden un homenaje oblicuo a generaciones anteriores o a otros cánones del pensamiento económico (Reinert, Ghosh y Kattel, 2016). El modelo es el de los vástagos neoclásicos -neoinstitucionalismo, nueva economía clásica, nuevo keynesianismo- cuya práctica vampírica coloniza palabras más antiguas y las vacía de su significado anterior. 
E1 dilema de estos vástagos radica en aceptar la falsa premisa de la ortodoxia a la que se propone servir como alternativa. La presunción es una búsqueda desapasionada de la verdad atemporal, "relajando los supuestos restrictivos" en aras de un "mayor realismo". Así, por ejemplo, en las teorías de la complejidad los agentes siguen reglas simples y terminan generando patrones intrincados e impredecibles, las funciones recursivas no lineales dan el mismo resultado, la varianza de los rendimientos resulta ser no normal, etc. Pero cuando el punto de partida es el modelo neoclásico de equilibrio general competitivo, estos ejercicios en gran medida pierden perspicacia y relevancia. Los teóricos del comportamiento pueden decirnos que la gente real no parece encajar bien en la descripción del "hombre económico" como buscador de placer autónomo, egoísta y obsesionado por las mercancías. Los teóricos de la complejidad pueden decirnos, como hace Arthur (2021), que un sistema construido a partir de invenciones de agentes que interactúan puede ser inestable. Incluso el observador menos atento del capitalismo real-existente ya sabía estas cosas ${ }^{3}$.

\section{ECONOMÍA EVOLUTIVA Y BIOFÍSICA}

El enfoque evolutivo y biofísico de los fenómenos económicos no es nuevo, y en realidad es muy anterior a la ortodoxia neoclásica de la que algunos creen que ha surgido. Comenzó con la interacción intelectual de Malthus y Darwin, se desarrolló a través de Marx y Henry Carey y (hasta cierto punto) en la obra de la escuela histórica alemana, elaborada y fermentada en la efervescencia pragmática y pluralista de la filosofía estadounidense de finales del siglo XIX, y logró una primera articulación completa en manos de Thorstein Veblen (1898). Después se desarrolló en la tradición institucionalista de John R. Commons (1934) y Clarence E. Ayres (1944), entre muchos otros, y fue la fuerza intelectual dominante en la economía estadounidense bajo el New Deal.

Las tradiciones keynesiana e institucionalista se fusionaron de nuevo en Norteamérica en la obra de John Kenneth Galbraith (Car-

${ }^{3}$ Es muy cierto que la aplicación de la física estadística a las finanzas reduce a escombros la teoría de las finanzas ortodoxas (Yakovenko y Rosser, 2021). Pero ¿qué añade eso a la experiencia de Long Term Capital Management (Galbraith, 2000), a la crisis asiática, a la crisis del NASDAQ, a la gran crisis financiera o incluso al Gran Crash de 1929 (Galbraith, 2009)? En particular, ¿qué sugieren estas nuevas teorías que hagamos? Un economista preocupado por la regulación efectiva del sistema bancario poco se beneficia de los enunciados matemáticos de la experiencia común.

Revista de Economía Institucional, vol. 24 , n. ${ }^{\circ} 46$, primer semestre/2022, Pp. $3^{-2} 5$ ISSN OI $24-5996 / \mathrm{E}-\mathrm{ISSN} 2346-2450$ 
ter, 2020), y la línea de trabajo post keynesiana fue continuada por Robert Eisner, Hyman Minsky, Paul Davidson y Wynne Godley. Hoy es popularizada, como teoría monetaria moderna, por William Mitchell, Randall Wray (2006), Stephanie Kelton (2020), Pavlina Tcherneva (2020) y otros autores. En Gran Bretaña, la causa keynesiana fue impulsada por Richard Kahn, Nicholas Kaldor (1985), Joan Robinson y otros, con estrechos vínculos con una versión italiana liderada por Luigi Pasinetti, Pierangelo Garegnani, Mario Nuti y otros. La calamidad de la gran crisis financiera se trata en muchos libros y artículos, un ejemplo notable es el de Varoufakis, Halevi y Theocarakis (2011). La atención específica al problema de la calidad de los recursos se originó en Jevons, fue desarrollada en la era moderna por Meadows et al. (1972) y hoy la prosigue la escuela biofísica (Hall y Klitgaard, 2018; Chen y Galbraith, 2009). Otra rama del enfoque institucionalista, con raíces en Marx y Keynes, apareció en Economía del Desarrollo, personificada por figuras como Albert Hirschman, Raúl Prebisch, Samir Amin y muchos otros, y hoy la llevan adelante Ha-Joon Chang e Ilene Grabel (2014), Jayati Ghosh y Luiz C. Bresser Pereira (2010), entre otros. Se podría identificar además una rama de estudios de la economía de transición y de China, en la que figura el nuevo pragmatismo de Grzegorz Kolodko (2020), junto con la historia pionera de Isabella Weber (2021) sobre la formulación de políticas en China. Hay muchos más; las aplicaciones varían según los problemas.

\section{EL ECONOMISTA ÚTIL}

La característica común de casi todas estas obras, excepto unas pocas dedicadas a escaramuzas lógicas con la ortodoxia neoclásica-como la controversia Cambridge-Cambridge sobre la teoría del capital (Robinson, 1956; Sraffa, 1960; Harcourt, 1972), o en microeconomía (Keen, 2011)- es que los protagonistas estaban preocupados ante todo por las cuestiones prácticas de política que enfrentaban sus gobiernos o la comunidad internacional de la que formaban parte. Fuese reformista o revolucionaria, su economía era (y sigue siendo) esclarecer los problemas y los medios para abordarlos. El propósito del razonamiento económico es informar y sostener opciones políticas y sociales. No se trata simplemente de hacer una simulación que emule de algún modo una serie de datos económicos.

El economista útil es aquel que se dedica a buscar soluciones. Un economista verdaderamente útil lo hace con mente abierta, de 
manera informada, consciente de los principios subyacentes, pero no hipnotizado por ellos, e independientemente de ganancias financieras y ambiciones personales, bien sean políticas o de estatus y celebridad entre los economistas. E1 comportamiento de los banqueros y especuladores, las emisiones de las fábricas y las redes de transporte, la extracción de recursos críticos de una reserva finita en la corteza de la tierra, el nivel y la distribución de los salarios, las ganancias y las rentas, la tributación justa y efectiva, cómo lograr la cooperación voluntaria de ciudadanos libres en la búsqueda del bien común: todo esto es parte de lo que un economista útil puede estudiar. La persona que se mantiene fuera y alejada de tales preguntas, que simplemente pretende "modelar el sistema" es, para la mayoría de los propósitos, un ocioso, no un científico sino un aficionado.

Así, el objetivo de Adam Smith era promover los intereses y el bienestar de la comunidad comercial de la que formaba parte, exponiendo las virtudes de los grandes mercados y la división del trabajo. David Ricardo intentó trasladar la carga tributaria de las ganancias a la renta, y Henry George intentó trasladarlos de los salarios a la renta, en ambos casos para que los impuestos recayeran sobre los terratenientes ociosos e improductivos. Karl Marx escribió El capital como fundamento teórico para la expropiación de los capitalistas. John Maynard Keynes intentó salvar y reformar a Gran Bretaña y al orden democrático burgués promoviendo una cura práctica para el desempleo masivo. John Kenneth Galbraith $(1958,1967)$ dirigió la atención de sus lectores a los problemas económicos de la abundancia: la miseria pública, la contaminación, la pobreza residual, el yermo cultural y estético, y el poder corporativo. Hyman Minsky describió las transiciones de fase de la inestabilidad financiera -cobertura, especulativa, Ponzi- y la necesidad de un gran gobierno y un gran banco central como dispositivos estabilizadores. Milton Friedman, un conservador comprometido, co-escribió una historia monetaria para respaldar las reglas monetarias (Friedman y Schwartz, 1963). En suma, la noción de que cualquier economista importante de cualquier siglo se ha mantenido al margen de las cuestiones de política de su época es mera pretensión.

\section{INVESTIGACIÓN ECONÓMICA}

Por tanto, la investigación económica, tal como debería ser, consiste en tratar de entender cómo funciona - $\mathrm{O}$ mal funciona- el complejo sistema en que vivimos en un momento determinado, y a qué tipo de 
fuerzas, presiones y políticas responde. Un ejemplo esclarecedor es la demostración de P. Chen (2021), a partir de datos reales, de que las crisis cambiarias "solo pueden ser causadas por oligarcas financieros". Otro fue el de Mandelbrot (1999), que mostró que el movimiento de precios de los activos de capital es bien modelado por un generador multifractal, por lo que está abierto a accidentes intrínsecamente impredecibles. Tales hallazgos tienen la propiedad de que se extraen de los fenómenos de la economía real existente, o se comparan directamente con ellos, de tal modo que motivan opciones políticas y sociales. No consisten en deducir políticas de primeros principios, ni en explorar las propiedades de sistemas matemáticos que -por interesantes que sean en sí mismos- se acercan mal o nada a la compleja economía en que vivimos. De nuevo, hay numerosos ejemplos de un buen trabajo; el problema no es que falte investigación sobre el mundo real entre los economistas y (en especial) entre científicos físicos que centran su atención en cuestiones económicas. Es más bien que esa investigación no tiene la posición que merece, porque no se puede integrar a la teoría dominante.

La siguiente sección argumenta que, para un mayor progreso, la economía para la era postneoliberal necesita desarrollar métodos empiricos de investigación adaptados a la perspectiva evolutiva, que hagan posible que los mundos de la academia y los de la política práctica vuelvan a asociarse de manera útil. Como escribió Peirce sobre Kepler, esto es lo que hay que hacer si se quiere mejorar la economía. E1 artículo presenta algunos enfoques extraídos de proyectos llevados a cabo por el autor durante cinco décadas. Se presentan aquí en parte con un espíritu de apología pro vita sua, pero también con la esperanza de que puedan iluminar en forma útil un argumento metodológico.

\section{EL PROBLEMA DE LA TAXONOMÍA ECONÓMICA}

Un problema característico en el análisis de sistemas complejos es la construcción de una taxonomía eficiente. Aquí es instructivo el ejemplo de la botánica. En manos de Linneo, se elaboró un hermoso sistema, una verdadera obra de arte, pero no ciencia en el sentido moderno. Hoy ya no se utiliza la clasificación de Linneo. En cambio, la taxonomía biológica se basa en la consanguinidad a nivel molecular, y refleja las divergencias del proceso evolutivo a lo largo del tiempo. En la clasificación de cualquier sistema complejo se aplican principios similares -de la química, la ingeniería y la antropología, y también se aplican a la historia de la tecnología (Basalla, 1989). Tales árboles 
evolutivos son fundamentales para la investigación científica acerca de cualquier campo complejo.

La economía, tanto en su encarnación académica como en su trabajo práctico, sigue ignorando en gran medida este requisito previo para la comprensión. La economía "puramente teórica” se caracteriza por taxonomías del tipo más primitivo e ideológico, que en gran parte reflejan las divisiones de clase reconocidas en Europa hace varios siglos (terratenientes, capitalistas, trabajadores) o sus remplazos desnaturalizados (capital y trabajo, hogares y empresas). La macroeconomía práctica se basa en la estructura taxonómica de las cuentas nacionales de ingreso y producto, que solo es comportamental en la medida en que Keynes (1936), Simon Kuznets, Richard Stone y otros arquitectos del sistema consideraron conveniente distinguir el consumo de los hogares, la inversión empresarial y el gasto público, así como las exportaciones y las importaciones, como categorías de comportamiento distintas. Casi un siglo después no es claro que esas distinciones sigan siendo válidas. Por ejemplo, el consumo de los hogares se compone de bienes no duraderos, duraderos y servicios. Pero mientras que el consumo de bienes no duraderos sigue de cerca al de servicios (hasta la pandemia), los bienes duraderos y la inversión empresarial comparten características. Por ello, un modelo de comportamiento podría reclasificar, en forma útil, los bienes duraderos de los hogares como una forma de inversión. Más en general, un análisis parsimonioso y eficiente del gasto agregado debería ir precedido de una reclasificación, de tal modo que las categorías taxonómicas no sean desdibujadas por patrones de comportamiento masivamente superpuestos, ni sean diferenciadas artificialmente por la fuerza del hábito. Pero en la literatura tales reclasificaciones preliminares del comportamiento de esquemas de categorías son raras o están ausentes.

Per contra, el análisis microeconómico, tiende a basarse en datos de encuestas, en general realizadas por un gobierno nacional en cumplimiento de una obligación accesoria, como un censo decenal o la Encuesta de Población Actual en Estados Unidos. Estas encuestas rara vez son idénticas o coordinadas entre países (con limitadas excepciones en la Europa moderna), por lo que compatibilizarlas para hacer comparaciones transnacionales es una tarea científica importante, emprendida en los últimos años para una gama limitada de países, en su mayoría ricos, por el Estudio de Ingresos de Luxemburgo. Pero hay una dificultad más profunda: la información recopilada está limitada por el mandato del encuestador, y se suele relacionar con características personales como el género, la edad, el origen étnico 
(como se define legalmente en el país), los años de escolaridad, etc. El resultado es una vasta literatura sobre la economía de la raza, el género y la educación, que presta mucha menos atención a cuestiones (como el cambio industrial) que no encajan fácilmente en la plantilla o no se registran como características de los individuos y los hogares.

En una línea similar, Thomas Piketty y sus colegas (Alvaredo et al., 2017) han explotado los registros del impuesto de renta para construir cuentas históricas de la distribución del ingreso en diversos países durante periodos que se extienden a más de un siglo en algunos casos. Este enfoque tiene ventajas sobre las encuestas en la medida en que los registros tributarios cubren un gran número de individuos y hogares, y parecen captar mejor la información de la cola superior de la distribución. Pero, igual que con las preguntas de encuestas o aún más, la información reportada es específica a nivel nacional, porque el ingreso gravable es un hecho legal del código tributario nacional, y los códigos tributarios varían ampliamente entre naciones. Además, el alcance general está limitado por el escaso mantenimiento de los registros, la elusión fiscal y el hecho de que muchos países no recaudan impuestos de renta (Galbraith, 2019b). Incluso en el caso de Estados Unidos, es esencial tener cuidado; los contribuyentes y los hogares no son categorías sinónimas (Rose, 2018), y los cambios en la legislación tributaria y en los incentivos para presentar la declaración pueden tener efectos adversos graves sobre la comparabilidad de los datos en el tiempo.

Otro tipo de estadísticas se refieren a empleadores, establecimientos, industrias y sectores, a menudo agrupadas por subdivisiones geográficas: estados, provincias, condados, municipios, etc. Estos datos son una reserva de información sobre lo que P. Chen (2021) siguiendo a Walt Rostow, denomina mesoeconomía, también conocida como estructura industrial o nivel de desarrollo económico. Pero estas medidas son característicamente bibliográficas y linneanas; las industrias y los sectores se agrupan conforme a una amplia y confusa variedad de criterios, que incluyen el tipo de producto, el tipo de proceso, la etapa del proceso de producción y otros. A veces surgen nuevas industrias y se agregan nuevas categorías o se subdividen las antiguas. El esquema de clasificación es jerárquico, igual que las subdivisiones geográficas las categorías se dividen y subdividen en capas de tamaño de grupo decreciente y detalle creciente. Pero las industrias y sectores así especificados son, hasta cierto punto, intrínsecamente arbitrarios; no rigen las similitudes subyacentes de genealogía o comportamiento y, por ello, cualquier estructura de grupo dada contendrá unidades cuya 
similitud o diferencia orgánica varía ampliamente. Como ocurre con casi todas las demás fuentes de datos, los economistas que trabajan en asuntos de política rara vez reconocen la cosificación de la estructura de categorías, lo que implica necesariamente aceptar una taxonomía anterior construida por no economistas para fines no relacionados. Una historia similar se aplica a las categorías del presupuesto en el análisis del gasto público; las categorías de gasto construidas por razones legales y políticas no son necesariamente informativas para el análisis social y político.

\section{CLASIFICACIÓN EVOLUTIVA EFICIENTE}

Este autor propuso un enfoque evolutivo de la taxonomía del presupuesto federal de Estados Unidos en una tesis doctoral (Galbraith, 1981), que luego desarrolló Berner (2005). También se elaboró y aplicó un enfoque paralelo a las industrias estadounidenses en Galbraith (1998), Ferguson y Galbraith (1999) y varios artículos incluidos en Galbraith y Berner (2001). En todos esos casos, la esencia era encontrar una variable de criterio adecuada y libre de unidades para medir las similitudes de comportamiento entre y dentro de las categorías taxonómicas. En el caso de las categorías del presupuesto, la variable es simplemente el cambio porcentual en el gasto nominal de un periodo (por lo general un año) al siguiente. Por tanto, cada categoría tiene un vector de características de longitud $\mathrm{T}-1$, donde $\mathrm{T}$ es el número total de periodos en el conjunto de datos. Una distancia euclidiana simple en el espacio ( $\mathrm{T}-1)$ da entonces una medida de la similitud de comportamiento, a partir de la cual se pueden construir clústeres que minimizan la varianza dentro del grupo, con un número de clústeres determinado por un criterio de pérdida de información a medida que avanza la agrupación escalonada.

En el caso de los datos industriales, el concepto de ingresos laborales específicos de la industria (Katz y Summers, 1989) hace posible utilizar los cambios en los salarios anuales promedio (técnicamente, nómina por empleado) como variable de criterio. Las categorías subyacentes pueden ser un conjunto único de datos jerárquicos por industria o región, o un híbrido de categorías, que incluyen el sector, la región, el género y otros, siempre que las categorías sean mutuamente excluyentes (no superpuestas). El árbol de clasificación resultante resume de manera eficiente las divergencias a través del tiempo, pues las entidades dentro de los clústeres no divergen o divergen menos que las entidades separadas en los diferentes niveles de ramificación del 
árbol. E1 árbol de clústeres es, por tanto, un mapa de la evolución de los elementos dentro de un sistema complejo. Después se elige una estructura de grupo adecuada por medio de una regla de detención: los grupos se conservan como entidades distintas, en vez de sumarse en etapas posteriores de la agrupación en clústeres, cuando la información perdida por la agrupación supera un umbral previamente especificado.

\section{EXTRAER INFORMACIÓN DE ESTRUCTURAS DE GRUPOS EVOLUTIVOS}

Una vez se logra una agrupación adecuada, un paso adicional es calcular las funciones discriminantes que representan la mayor proporción de variación entre grupos. Estas funciones son un vector de coeficientes ponderados (vectores propios) de la matriz de vectores de series de tiempo que sustentan el esquema de categorías evolutivas construido. Los vectores propios resultantes son en sí mismos variables sintéticas de series de tiempo, que captan las fuerzas que mueven la variación entre grupos. Los valores propios correspondientes dan el peso relativo o la importancia de cada fuerza en la contabilidad de las variaciones entre grupos. Las gráficas de los productos cruzados resultantes ilustran la proximidad y la distancia de los elementos subyacentes a lo largo de las diversas dimensiones. Como paso final, cada vector propio se puede comparar con series de tiempo históricas para identificar las fuerzas económicas, políticas y sociales en juego. Para una presentación completa de la técnica, ver Galbraith y Lu (1999).

De este modo, Ferguson y Galbraith (1999) demostraron que los cambios salariales relativos entre 1920 y 1946 en Estados Unidos fueron impulsados por cambios en la demanda efectiva, la organización laboral y la actividad huelguística, y los movimientos de la tasa de cambio, en ese orden de importancia, que en conjunto representaron el $90 \%$ de los efectos diferenciales significativos. Este análisis obvió entonces los efectos hipotéticos de los niveles de educación, la demanda de habilidades, las nuevas tecnologías, etc., comúnmente mencionados en la literatura convencional, en gran parte por motivos a priori (Goldin y Katz, 2010). Galbraith (1998) hizo un análisis similar para Estados Unidos entre 1958 y 1992, que identificó las variaciones en la inversión empresarial, el gasto de consumo, la protección comercial y la guerra como cuatro fuerzas que representan un 59\% de la variación de los salarios entre industrias.

Así, la técnica es no paramétrica y ateórica, pero capaz de rastrear con alta precisión las condiciones cambiantes de un sistema econó- 
mico complejo y de una manera que aclara el impacto de las políticas, las movilizaciones masivas, los mercados externos y las condiciones ambientales en los resultados distributivos.

\section{COMPLEJIDAD Y PATRONES RELEVANTES DE POLÍTICA: EL CASO DE LAS DESIGUALDADES}

Los sistemas económicos reales existentes tienen propiedades que son aclaradas por el comportamiento de funciones recursivas no lineales simples; en particular, muestran transiciones de fase -la tricotomía de Minsky de las posiciones financieras (cobertura, especulativa y Ponzi) es un ejemplo (Minsky, 2008) - y características de los sistemas producidos por generadores multifractal, en particular distribuciones de cambios de precios de los activos con varianza infinita y una tendencia al colapso repentino e imprevisto. Estas son heurísticas útiles, que indican en particular la utilidad de los límites al comercio, de los interruptores del circuito, de los controles de precios (Galbraith, 1952) y de los sistemas de almacenaje-desalmacenaje de productos básicos clave (Graham, 1997). Esas políticas se han empleado desde la antigüedad para estabilizar economías inestables (Weber, 2021).

Las propiedades fractales y las autosimilitudes de las economías reales ofrecen otra oportunidad de investigación relevante para las políticas: aprovechar lo que es visible y está registrado para medir lo que es parcialmente invisible y no está registrado. Una característica de los conjuntos de datos administrativos - de nuevo, por sector, industria o región- es que se recogen de manera rutinaria, en un formato estable y con regularidad, compilando un registro consistente en el tiempo y en el espacio. Su cobertura es sesgada, por supuesto: no cubren el trabajo informal, y suelen cubrir mal los servicios y la agricultura. Pero la autosimilitud sugiere, y a veces dictamina, que las fluctuaciones observadas entre las categorías y los grupos cuyo tamaño e ingresos promedio se miden en los datos tendrán una relación consistente con los sectores no observados de la economía compleja.

Así, la evolución de una medida de desigualdad entre grupos, usualmente el componente entre grupos del estadístico $\mathrm{T}$ de Theil (Theil, 1972; Galbraith, 2014), captará los principales movimientos de la desigualdad en la economía en su conjunto. Para una discusión completa de la teoría, ver Conceição et al. (2001). Y una compilación de tales medidas permite elaborar medidas densas y consistentes de desigualdad entre países y regiones que cubren extensos periodos 
históricos, junto con detalles precisos sobre los grupos (regiones, sectores, industrias) que impulsan el cambio de las medidas generales (Galbraith y Kum, 2005). De ese modo, se hace posible una nueva explicación del cambio estructural complejo. Para más detalles de los conjuntos de datos de desigualdad global, su calidad y sus usos, ver Galbraith, Halbach et al. (2016) y Galbraith, Ghoi et al. (2016).

Después de elaborar un panel de medidas de desigualdad apropiadamente denso y consistente, la simple aplicación de una regresión bidireccional de efectos fijos permite hacer una descomposición bidimensional, que produce una clasificación consistente de las desigualdades entre países (u otras unidades geográficas), y un mapeo del patrón común de cambio en el tiempo (Galbraith y Ghoi, 2020). Surge entonces una macroeconomía de las desigualdades a nivel global (Galbraith, 2007, 2019), Los patrones de cambio de esos datos para el periodo que se inició desde comienzos de los años sesenta revelan claros puntos de inflexión, que corresponden a la crisis financiera global de principios de los años ochenta y al pico del boom de crédito de 2000-2001, y ponen de relieve en forma contundente el papel de la deuda, las tasas de interés y las crisis financieras como impulsores de la desigualdad económica en la economía mundial. Esto, a su vez, apunta directamente hacia políticas relevantes a escala global.

La integración de los resultados distributivos y las fuerzas que afectan a la economía en su conjunto aclara la necesidad de romper otro mal hábito taxonómico profundamente arraigado: la distinción entre "macro" y "micro" economía. Esta distinción surgió como un compromiso político en los departamentos de economía estadounidenses después de la Segunda Guerra Mundial, entre los keynesianos temporalmente en ascenso y los grandes estratos de "pequeños pensadores decididos" (Solow, 1967) formados en el análisis marshalliano de oferta y demanda, que no eran capaces ni estaban dispuestos a dar el salto de la mecánica neoclásica newtoniana a la apelación de Keynes a la relatividad de Einstein como base de una teoría integrada de la economía (Galbraith, 1996). Pero la demostración empírica de que los cambios en la distribución -el principal tema del análisis microeconómico- son impulsados por un pequeño número de grandes fuerzas que actúan en el tiempo sobre el conjunto de la economía es determinante para un cambio de teoría.

De manera similar la demostración empírica de que las economías nacionales están estrechamente vinculadas -y no solo en Europa, donde la integración política de facto está muy avanzada- aboga por un análisis económico global integrado como punto de partida del 
pensamiento económico. El hecho de que las agencias de estadísticas operen principalmente en otros niveles es un inconveniente, pero no una excusa.

\section{LA REGULACIÓN: UN RETO POLÍTICO GENERAL DE LAS ECONOMÍAS REALES}

La complejidad que surge en sistemas abiertos y disipativos (Chen, 2021) como parte del proceso de la vida no es economía. Es una idea universal que se basa en la física e ilumina por igual los sistemas biológicos, mecánicos y sociales. Una característica común a todos estos sistemas es la regulación; la mecánica de la supervivencia exige que las fuerzas que atraviesan el sistema estén contenidas -en términos de temperatura, presión, volumen- dentro de la capacidad de los materiales con los que se construye el sistema para que puedan soportarlas (Chen y Galbraith, 2011, 2012a y 2012b). Una economía posneoliberal apropiada es el arte de aplicar este principio al funcionamiento de la vida económica. A veces, esto implica levantar restricciones que ya no son necesarias; otras, crear e imponer regulaciones y normas que fomenten la estabilidad, la sostenibilidad y la resiliencia.

En particular, la inestabilidad financiera, apuntalada por la fuerte tendencia de los mercados financieros libres a degenerar en oleadas de fraudes financieros, es una fuerza impulsora clave de la crisis, del colapso y del aumento de las desigualdades a nivel global. El problema para el economista político es entonces muy claro: ¿cómo estabilizar el sector financiero global? Ese problema no es nuevo; se abordó con gran fuerza en Estados Unidos a inicios de los años treinta con la Ley Bancaria de Emergencia, la creación de la Comisión de Bolsa y Valores, la separación de la banca de inversión y la banca comercial y la introducción del seguro federal de depósitos. Además, es evidente que la desregulación del sector financiero, impulsada en Estados Unidos desde finales de los años setenta y emulada en todo el mundo, propició el resurgimiento de la inestabilidad y la última crisis. Por ello, la atención se centra en cómo lograr una re-regulación adecuada y reafirmar el control estabilizador, sin suprimir al mismo tiempo las funciones legítimas del crédito y la deuda.

El problema de una regulación financiera adecuada, eficaz y autónoma a escala global es uno de los más difíciles que hoy enfrenta el economista político, pero su propósito aquí es ilustrar un caso del problema de política general: cuál es la mejor manera de regular el sistema económico. En la necesidad de regulación, los sistemas económicos no difieren de los sistemas biológicos o mecánicos; sin regulación, mantenimiento y reglas de circulación, invariablemente 
fallan en poco tiempo. Al comprender la naturaleza y el propósito de la regulación, llegamos a una diferencia básica entre los economistas reales y sus simulacros académicos dominantes, ortodoxos y orientados a los modelos.

Según la corriente dominante, la economía pura es un mundo autorregulado; el único requisito para alcanzar el equilibrio con el máximo bienestar social es asignar todos los derechos de propiedad y que el sistema de precios tenga total libertad para ajustarse. Todo impedimento para el resultado óptimo se debe a externalidades, distorsiones e intervenciones, y la función del economista es intentar eliminarlas en la medida de lo posible. Esta mentalidad ayuda a explicar, por ejemplo, el entusiasmo de algunos economistas por la pequeña empresa, su hostilidad hacia los sindicatos y los impuestos, y la recurrente invocación a la competencia como medio para garantizar un mejor desempeño económico. La regulación es, entonces, un enfoque de segundo óptimo, que tendría costos y beneficios, y que solo se debe imponer en el grado mínimo necesario para compensar los impedimentos a la optimización que no se pueden eliminar.

Para el economista que trabaja en políticas del mundo real, la regulación no es un complemento, es una condición necesaria para que surjan estructuras complejas. La regulación es el conjunto de leyes, reglas, normas y hábitos que hacen posible el funcionamiento sostenido de sistemas complejos. Solo la economía de Robinson Crusoe, que carece de una sociedad real, puede prescindir de ella, y únicamente en ausencia de limitaciones de recursos o ambientales, que afectan incluso la sostenibilidad de Robinson Crusoe en su isla a lo largo del tiempo. En el mundo real, sin regulación económica no habría largas cadenas de producción, ni líneas de crédito estables, ni confianza en los supermercados o los electrodomésticos o los medicamentos, ni viajes aéreos, ni mercado masivo de automóviles o de cualquier otro aparato complejo. De hecho, el proceso de desarrollo económico se puede definir razonablemente como el logro de normas reguladoras que permitan el surgimiento de actividades económicas complejas a gran escala y su mantenimiento en el tiempo. Los países ricos tienen estas normas y -si quieren seguir siendo ricos- las hacen cumplir.

\section{CONCLUSIÓN}

Está fuera de toda duda que la economía mundial es un sistema complejo. Para los economistas, el problema es cómo afrontar de la 
mejor manera esta realidad. Un enfoque popular es empezar con las simplicidades premodernas del modelo neoclásico, y mostrar que se producen diferencias fundamentales en el comportamiento del modelo cuando se relajan los supuestos más elementales. Este es un progreso del tipo más limitado, que proporciona cierta sensación de logro intelectual, pero ninguna guía real al economista, cuya tarea es ayudar a la sociedad a pasar del presente al futuro.

La alternativa, defendida y descrita en este artículo, es explotar los métodos de la ciencia evolutiva y algunas propiedades de los sistemas complejos para clasificar, medir, analizar y entender las fuerzas que impulsan los cambios económicos significativos a nivel global, continental, nacional y local. Este es el tipo de conocimiento que luego se puede convertir en la tarea práctica de la gobernanza económica, en la búsqueda de valores comunes para la sociedad en su conjunto: seguridad, sostenibilidad, prosperidad y libertad. Aunque los métodos evolucionarán con las circunstancias, este es, en términos generales, el enfoque que han adoptado todos los economistas de la historia cuyo nombre quizá sea recordado.

\section{REFERENCIAS BIBLIOGRÁFICAS}

Alvaredo, F., Chancel, L., Piketty, T. et al. (2017). World inequality report 2018. Paris: World Inequality Lab.

Arrow, K. y Debreu, G. (1954). Existence of an equilibrium for a competitive economy. Econometrica, 22(3), 265-290.

Arthur, B. (en preparación). Foundations of complexity economics. En P. Chen, W. Elsner y A. Pyka (eds.), A handbook of complexity economics. Nueva York: Routledge.

Ayres, C. E. (1944). The theory of economic progress. Chapel Hill: University of North Carolina Press.

Azmanova, A. (2020). Capitalism on edge: How fighting precarity can achieve radical change without crisis or utopia. Nueva York: Columbia University Press.

Basalla, G. (1989). The evolution of technology. Cambridge: Cambridge University Press.

Berner, M. (2005). The federal budget's core and periphery: Patterns of budget outcomes. Journal of Public Budgeting, Accounting and Financial Management, 17(4), 437-470.

Black, W. K. (2005). The best way to rob a bank is to own one: How corporate executives and politicians looted the SEL Industry. Austin: University of Texas Press.

Bresser P., L. C. (2010). Globalization and competition: Why some emergent countries succeed while others fall behind. Cambridge: Cambridge University Press.

Carter, Z. D. (2020). The price of peace: Money, democracy and the life of John Maynard Keynes. Nueva York: Random House. 
Chang H. y Grabel I. (2014). Reclaiming development: An alternative economic policy manual. Londres y Nueva York: Zed Books.

Chen P. (en preparación). From complexity science to complexity economics. En P. Chen, W. Elsner y A. Pyka (eds.), A handbook of complexity economics. Nueva York: Routledge.

Chen, J. y Galbraith, James K. (2009). A biophysical approach to production theory, UTIP working paper No. 55, [http://utip.lbj.utexas.edu].

Chen, J. y Galbraith, James K. (2011). Institutional structures and policies in an environment of increasingly scarce and expensive resources: A fixed cost perspective. Journal of Economic Issues, 45(2), 301-309.

Chen, J. y Galbraith, James K. (2012a). A common framework for evolutionary and institutional economics. Journal of Economic Issues, 46(2), 419-428.

Chen, J. y Galbraith, James K. (2012b). Austerity and fraud under different structures of technology and resource abundance. Cambridge Journal of Economics, 36(1), 335-343.

Commons, J. R. (1934). Institutional economics. Nueva York: Macmillan.

Conceição, P., Galbraith, James K. y Bradford, P. (2001). The Theil Index in sequences of nested and hierarchical grouping structures: Implications for the measurement of inequality through time, with data aggregated at different levels of industrial classification. Eastern Economic Journal, 27(4), 491-514.

Davis, W. W. (1983). "China, the Confucian ideal, and the European Age of Enlightenment. Journal of the History of Ideas, 44(4), 523-548.

Ferguson, T., Galbraith, James K. (1999). The American wage structure, 1920-1947. Research in Economic History, 19, 205-257

Frank, T. (2020). The people, no: A brief history of anti-Populism. Nueva York: Metropolitan Books.

Friedman, M. y Schwartz, A. J. (1963). A monetary history of the United States. Princeton: Princeton University Press.

Fukuyama, F. (1992). The end of history and the last man. Nueva York: Free Press.

Gaffney, M. (2007). Neo-classical economics as a stratagem against Henry George. En M. Gaffney y F. Harrison (eds.), The corruption of economics (pp. 29-164). Londres: Shepheard-Walwyn Publishing Co.

Galbraith, J. K. (1952). A theory of price control. Cambridge: Harvard University Press.

Galbraith, J. K. (1958). The affluent society. Cambridge, MA: Houghton-Mifflin.

Galbraith, J. K. (1967). The new industrial State. Cambridge, MA: HoughtonMifflin.

Galbraith, J. K. (2009). The great crash, 1929. Nueva York: Mariner Books.

Galbraith, James K. (1981). A theory of the government budget process, PhD Dissertation submitted to the Economics Department, Yale University.

Galbraith, James K. (1996). Keynes, Einstein and scientific revolution. En P. Arestis y M. Sawyer (eds.), Keynes, money and the open economy: Essays in honor of Paul Davidson, v. 1 (pp. 14-21). Aldershot: Edward Elgar.

Galbraith, James K. (1998). Created unequal: The crisis in American pay. Nueva York: The Free Press. 
Galbraith, James K. (2000). Capital mismanagement: Roger Lowenstein, when genius failed: The rise and fall of long-term capital management. The Washington Monthly, 32(9), 46-47.

Galbraith, James K. (2007). Global inequality and global macroeconomics. Journal of Policy Modeling, 29(4), 587-607.

Galbraith, James K. (2014). Inequality and instability: A study of the world economy just before the Great Crisis. Nueva York: Oxford University Press.

Galbraith, James K. (2019a). A global macroeconomics - yes, macroeconomics, dammit - of inequality and income distribution. Review of Keynesian Economics, 7(1), 1-5.

Galbraith, James K. (2019b). Sparse, inconsistent and unreliable: Tax records and the World Inequality Report 2018. Development and Change, 50(2), 329-346.

Galbraith, James K. (2020). Bad economic theory and practice, demolished. The American Prospect, [https://prospect.org/economy/bad-economictheory-and-practice-demolished/].

Galbraith, James K. y Berner, M. (eds.). (2001). Inequality and industrial change: A global view. Nueva York: Cambridge University Press.

Galbraith, James K. y Choi, J. (2020). Inequality under globalization: State of knowledge and implications for economics. Real-World Economics Review, 92, 84-102.

Galbraith, James K. y H. Kum, (2005). Estimating the inequality of household incomes: Toward a dense and consistent global data set. Review of Income and Wealth, 51(1), 115-143.

Galbraith, James K. y Lu, J. (2001). Cluster and discriminant analysis on time-series as a research tool. UTIP, documento de trabajo $N^{o}$. 6, [http:// utip.lbj.utexas.edu].

Galbraith, James K., Choi, J., Halbach, B. et al. (2016). A comparison of major world inequality data sets: LIS, OECD, EU-SILC, WDI and EHII. En L. Cappellari, S. W. Polachek y K. Tatsiramos (eds.), Income inequality around the world. Research in labor economics, v. 44 (pp. 1- 48). Bingley: Emerald Group.

Galbraith, James K., Halbach, B., Malinowska, A. et al. (2016). The UTIP global inequality data sets 1963-2008: Updates, revisions and quality checks. En K. Basu, V. Hon y J. Stiglitz (eds.), Inequality and growth (pp. 87-106). Basingstoke: Palgrave MacMillan.

Georgescu-R. N. (1971). The entropy law and the economic process. Cambridge: Harvard University Press.

Goldin, C., Katz, L. (2010). The race between technology and education. Cambridge: Harvard University Press.

Graham, B. (1997). Storage and stability: A modern ever-normal granary. Nueva York: McGraw-Hill.

Hall P. A. y Soskice D. (2001). Varieties of capitalism: The institutional foundations of comparative advantage. Oxford y Nueva York: Oxford University Press.

Hall, C. A. S. y Klitgaard, K. (2018). Energy and the wealth of nations: An introduction to biophysical economics. Cham: Springer International Publications. 
Harcourt, G. (1972). Some Cambridge controversies in the theory of capital. Cambridge: Cambridge University Press.

Kaldor, N. (1985). Economics without equilibrium. Armonk: M. E. Sharpe. Katz, L. y Summers, L. (1989). Industry rents: Evidence and implications. Brookings Papers on Economic Activity: Microeconomics, 1, 209-290.

Keen, S. (2011). Debunking economics: The naked emperor dethroned? Nueva York: Zed Books.

Kelton, S. (2020). The deficit myth: Modern monetary theory and the birth of the people's economy. Nueva York: Public Affairs.

Keynes, J.M. (1936). The general theory of employment, interest and money. Londres: MacMillan.

Kolodko, G. (2020). China and the future of globalization: The political economy of China's rise. Londres y Nueva York: I.B. Tauris.

Mandelbrot, B.B. (1999). A multifractal walk down Wall Street. Scientific American, 280(2), 70-73.

Meadows, D. H., Meadows, D. L., Randers, J. et al. (1972). The limits to growth: A report for the Club of Rome's Project on the predicament of mankind. Nueva York: Universe Books.

Minsky, H. (2008). Stabilizng an unstable economy. Nueva York: McGrawHill Educational.

Peirce, C. S. (1877). Illustrations of the logic of science. The Popular Science Monthly, XII, p. 2.

Reinert, E., Ghosh, J. y Kattel, R. (2016). Handbook of alternative theories of economic development. Cheltenham: Edward Elgar.

Robinson, J. (1956). The accumulation of capital. Londres: MacMillan.

Rose, S. (2018). Parsing Piketty: Getting the facts right on inequality and middle-class incomes. Manuscrito inédito.

Samuelson, P. A. (1947). Foundations of economic analysis. Cambridge, MA: Harvard University Press.

Samuelson, P.A. (1948). Economics. Nueva York: McGraw-Hill.

Scarf, H.E. y Hansen, T. (1973). The computation of economic equilibria. Cowles Foundation, Monograph No. 24, New Haven, CT y Londres, UK: Yale University Press.

Schumpeter, J.A. (1942). Capitalism, socialism and democracy. Nueva York: Harper and Brothers.

Smith, A. (1776). An inquiry into the nature and causes of the wealth of nations. Edimburgo, disponible en línea y en muchas ediciones.

Solow, R. (1967). The new industrial State: Son of affluence. The Public Interest, [https://www.nationalaffairs.com/public_interest/detail/the-newindustrial-state-son-of-affluence].

Sraffa, P. (1960). Production of commodities by means of commodities. Cambridge: Cambridge University Press.

Tcherneva, P. (2020). The case for a job guarantee. Nueva York: Polity.

Theil, H. (1972). Statistical decomposition analysis: With applications in the social and administrative sciences. Ámsterdam y Londres: North-Holland.

Varoufakis, Y., Halevi, J. y Theocarakis, N. (2011). Modern political economics: Making sense of the post-2008 world. Nueva York: Routledge.

Veblen, T. (1898). Why is economics not an evolutionary science? The Quarterly Journal of Economics, 12(4), 373-397. 
Weber, I.M. (2021). How China escaped shock therapy. Nueva York: Routledge. Wray, R. (2006). Understanding modern money: The key to full employment and price stability. Cheltelham: Edward Elgar.

Yakovenko, N. y Rosser, B. (en preparación). Statistical mechanics of money, wealth and income. En P. Chen, W. Elsner y A. Pyka (eds.), A handbook of complexity economics. Nueva York: Routledge. 